\title{
Combination Therapy in Antihypertensive Treatment
}

\section{Abstract}

Antihypertensive treatment is associated with risk reduction of cardiovascular events like coronary heart disease, heart failure, stroke and renal events. Monotherapy is indicated in the treatment algorithm especially for grade 1 or mild hypertension. In the vast majority of hypertensive patients, effective BP control can only be achieved by combination of at least two antihypertensive drugs. Combination antihypertensive therapy is recommended in recent guidelines as a potential first-line therapy in patients with high baseline blood pressure and high cardiovascular risk. The combination therapy consisting of ACE inhibitor and diuretic or ACE inhibitor and calcium antagonist are recommended and effective drug combinations in major clinical trials.

Keywords: Cardiovascular events; Antihypertensive treatment; Combination therapy

\author{
Mini Review \\ Volume 5 Issue 1 - 2016 \\ Otljanska Magdalena* and Boshev Marjan \\ University Clinic of Cardiology, Medical Faculty, University \\ "Sts. Cyril and Methodius", Macedonia \\ *Corresponding author: Otljanska Magdalena, University \\ Clinic of Cardiology, Medical Faculty, University Campus \\ "Mother Theresa" - Skopje, Republic of Macedonia, Tel: +389 \\ 72231 006; Email: magdalenaotljanska@gmail.com
}

Received: January 17, 2016 | Published: January 22, 2016

\section{Introduction}

One of the most prevalent and important disease worldwide is arterial hypertension with prevalence of $35-40 \%$. When we look worldwide prevalence of hypertension in 2000 , we can see that approximately 972 millions of people were with hypertension, and the estimation is that around 1560 millions of people in 2025 will have hypertension [1]. There is a linear association between increased blood pressure and risk of cardiovascular events. High blood pressure is a strong, major risk factor for coronary artery disease, heart failure, stroke, renal disease, as well as peripheral arterial disease and it is a significant contributor to global mortality. Benefits of lowering blood pressure by approximately 12/4-5 mm Hg (SBP/DBP) will reduce stroke incidence by $35-$ $40 \%$, myocardial infarction by $20-25 \%$, and heart failure by $50 \%$ or even small reductions in blood pressure can reduce risk of cardiovascular mortality. Only $2 \mathrm{~mm} \mathrm{Hg}$ decrease in mean office systolic blood pressure reduces stroke and ischemic heart disease mortality risk by $10 \%$ and $7 \%$, respectively.

Goals of antihypertensive treatment include not only reduction of high blood pressure but also prevention of target organ damage, as well as reduction of hypertension-associated cardiovascular morbidity and mortality to achieve short- and long-term prevention of cardiovascular events (Figure 1).

From the European guidelines on hypertension 2013, the choice of antihypertensive drugs - there are five major antihypertensive drugs classes - diuretics, ACE inhibitors (ACE-I), calcium antagonists (CA), angiotensin-receptor blockers (ARB), and B-blockers - do not differ significantly in their overall ability to lower blood pressure in hypertension, but major drug classes differ in their ability to protect against overall cardiovascular risk of cause-specific cardiovascular events, such as stroke and myocardial infarction. Despite the availability of different classes of antihypertensive drugs, blood pressure is suboptimally controlled in majority of hypertensive patients, only 20-30\% have blood pressure $<140 / 90 \mathrm{~mm} \mathrm{Hg}$. Non-adherence is one of the important reasons for poor control of blood pressure in hypertensive patients, and a therapy consisting of too many tablets per day is one of the reasons for patient non-compliance. There are patients with good compliance and patients with poor compliance depending on the number of tablets. Most patients cannot be managed by monotherapy. Monotherapy is indicated in the treatment algorithm especially for grade 1 or mild hypertension. In the vast majority of hypertensive patients effective blood pressure control can only be achieved by combination of at least two antihypertensive drugs. Recommended combinations for priority use are diuretic + ACE-I, diuretic + ARB, diuretic + CA, ACE-I + CA, and ARB +CA [2,3].

Major clinical studies have shown that patients generally need an average of 2 antihypertensive drugs to achieve target blood pressure. Percentage of patients requiring $\geq 2$ antihypertensive drugs to reach blood pressure targets for example in LIFE Study was $90 \%$, in ALLHAT Study - $63 \%$ and $78 \%$ of patients in ASCOT Study. Patients with diabetes or renal impairment required an average of 3,2 blood pressure medications to reach lower blood pressure goal.

There are many advantages of combination over monotherapy for hypertension control: faster achievement of target blood pressure with greater efficacy and higher response rates, improved outcomes, potential for fewer side effects, increased potential for end-organ protection, as well as simple and convenient regimen which improves patient compliance.

However, combination of two or more drugs must be logical with complementary and synergistic pharmacodynamics of individual components and pharmacokinetic properties with once-daily dosing. Low-dose combination therapy may have greater cardiovascular benefits than titration of monotherapy to higher doses and less probability of side effects of the drugs. We consider two-drug combination therapy in patient with: high baseline blood pressure and high cardiovascular risk. Combination therapy with fixed doses of two drugs in a single tablet may be recommended due to potential for improved adherence. Combination treatment leads to a greater blood 
pressure reduction and faster blood pressure lowering to target levels, prompter response in a larger number of patients, lower probability of discouraging patient adherence with many treatment changes and reduced risk of hospitalization due to cerebrovascular and coronary events in about 25\%.

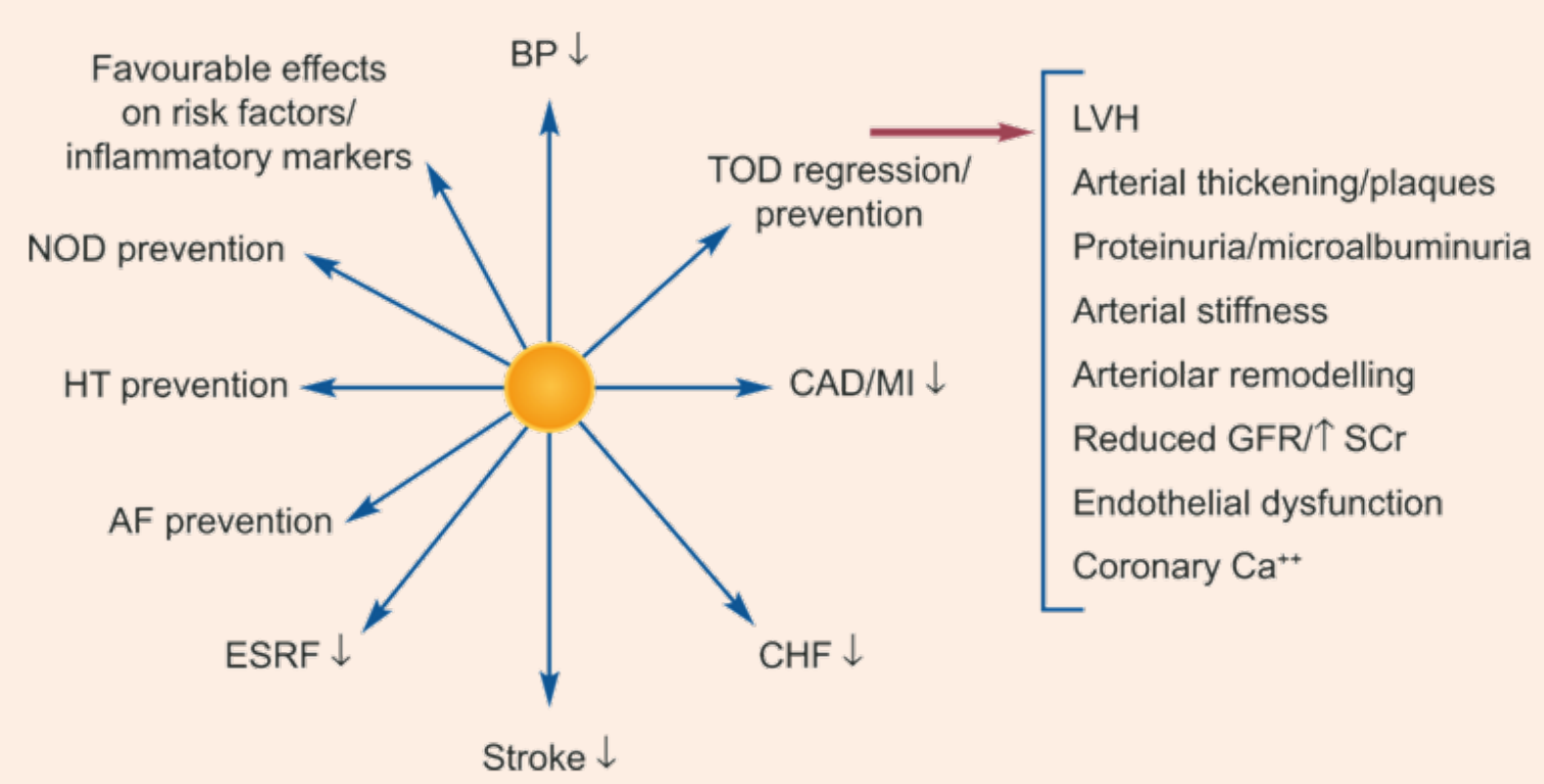

Figure 1: Goals of antihypertensive therapy.

The use of fixed-dose combination or flexible dose combination can simplify the treatment. Combination of ACE-I and CA can be first choice of treatment for hypertension. Perindopril has some advantages over other ACE-I: long-lasting effect (24-hour blood pressure control), one of the most studied ACE-Is, reduces atherosclerotic disease progression, improves endothelial dysfunction, has antithrombotic and anti-inflammatory effect. Amlodipin has long-lasting effect (24-hour blood pressure control), no need for caution and dose adjustment when combined with a beta-blocker, has antioxidative effect, it is dihydropyridine calcium channel blocker with the widest indications, the calcium channel blocker available in the greatest variety of FDCs with other active substances. With just one tablet a day we achieve effective blood pressure lowering, $24 \mathrm{~h}$ blood pressure control and improved patient compliance. The combination of perindopril and amlodipine has a complementary mechanism of action which leads to less side effects of amlodipine, widening the arteries and veins and reduced ankle edema $[4,5]$.

Combination of perindopril and amlodipine reduces the incidence of all cardiovascular events in patients with hypertension and the most common risk factors - type 2 diabetic patients (13\%), patients with metabolic syndrome (16\%), smokers (23\%), patients with previous vascular diseases (20\%), and patients with renal impairment (17\%) according to ASCOT Study. In this study, combination of perindopril and amlodipine reduces all important $\mathrm{CV}$ events and total mortality in hypertensive high risk patients total mortality by $11 \%$, cardiovascular mortality by $24 \%$, stroke by $23 \%$, new-onset diabetes by $31 \%$, renal impairment by $15 \%$ and total coronary endpoint by $13 \%$.
There is a novel fixed dose combination of $2,5 \mathrm{mg}$ amlodipine and 3,5 mg perindopril once daily for first-step treatment in patients with uncomplicated mild-to-moderate hypertension. The combination gave the similar blood pressure reduction to amlodipine $5 \mathrm{mg}$ but with better tolerability profile. Perindopril/ indapamide is useful drug combination in cardiovascular continuum (Figure 2). Indapamide has some advantages over hydrohlortiazide: it is metabolically neutral, protects against stroke, protects the heart, and reduces total mortality.

Blood pressure treatment in the very elderly, i.e. aged over 80 suggests that blood pressure lowering reduces the risk of stroke, heart failure and death in people aged over 80 years as in HYVET Study. This trial investigated the effects of placebo-controlled blood pressure lowering, using perindopril and indapamide in very elderly patients and beside effects of reducing blood pressure, this combination therapy reduced all-cause mortality by $21 \%$, cardiovascular mortality by $23 \%$ and stroke mortality by 39\% compared with placebo treatment [5-7].

The PARAMETER Study (Prospective comparison of Angiotensin Receptor neprilysin inhibitor with Angotensin Receptor Blockers measuring arterial stiffness in the elderly) demonstrated the ability of LCZ696 to reduce central and pulse pressure more effectively than ARB in high risk elderly patients with systolic hypertension. The results of this study provide therapeutic advantage of LCZ696 in older hypertensive patients with stiff arteries, reducing central aortic systolic and pulse pressure more than ARB. 


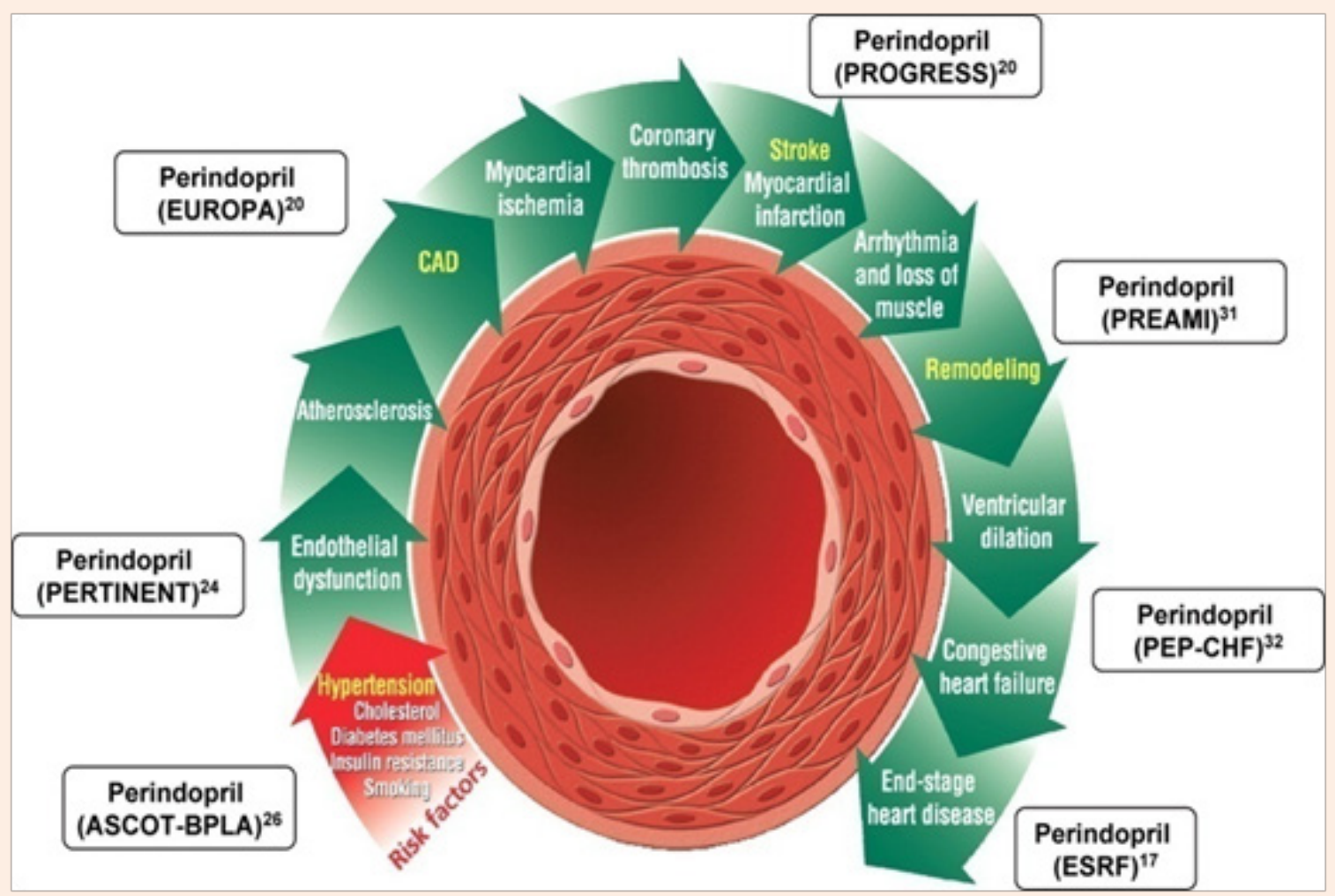

Figure 2: Perindopril in cardiovascular continuum.

PROGRESS - perindopril and indapamide protection against recurrent stroke study (6105 pts) in secondary prevention trial active treatment reduce the risk of stroke by $43 \%$ and reduction in mortality by $28 \%$.

EUROPA - EUropean trial on Reduction Of cardiac events with Perindopril among patients with stable coronary Artery disease (12218 pts). Combination of perindopril and calcium channel blocker reduces risk of death in patients with stable coronary heart disease.

PERFECT- (PERindopril-Function of the Endothelium in Coronary artery disease Trial) and PERTINENT - (Perindopril-thrombosis, Inflammation, endothelial dysfunction, and neurohormonal activation trial) are substudy of EUROPA Study.

ADVANCE Study - in this study perindopril/indapamide reduce $18 \%$ cardiovascular mortality, $14 \%$ all cause mortality and $21 \%$ microalbuminuria (11140 pts).

\section{Conclusion}

These are some of the studies with combination therapy in antihypertensive treatment and benefits of reduction of cardiovascular events and mortality beside reduction of blood pressure. Dual combination of perindopril/amlodipine and perindopril/indapamide has been documented as effective in reducing blood pressure with improved cardiovascular events and reduced total mortality. The combination of new drugs will prove the benefit of cardiovascular protection in hypertensive patients.

\section{References}

1. Kearney PM, Whelton M, Reynolds K, Muntner P, Whelton PK, et al. (2005) Global burden of hypertension:analysis of worldwide data. Lancet 365(9455): 217-223.Duprez DA (2006) Role of the renin-angiotensin-aldosterone system in vascular remodeling and inflammation: A clinical review. J Hypertens 24(6): 983-991.
2. Mancia G, Fagard R, Narkiewicz K, Redón J, Zanchetti A, et al. (2013) ESH/ESC Guidelines for the management of arterial hypertension. The Task Force for the Management of Arterial Hypertension of the European Society of Hypertension (ESH) and of the European Society of Cardiology (ESC). J Hypertension 31(7): 1281-1357.

3. Elliot W (2002) Is fixed dose combination therapy appropriate for initial hypertensive treatment? Curr Hypertens Rep 4(4): 278-285.

4. Poulter N (2014) Multiple Combination Therapy in Hypertension.

5. Messerli F (2003) Combination Therapy and Hypertension. Science Press.

6. Schmieder E (2013) Why do we need antihypertensive combinations? Medicographia 35(4): 117. 